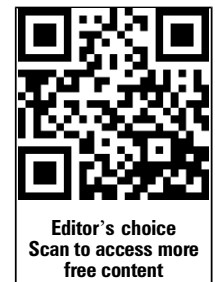

1 Department of Tropical

Medicine at the Bernhard, Nocht Institute, German Armed Forces Hospital Hamburg, Hamburg, Germany

${ }^{2}$ Institue for Forensic Medicine, University Hospital Eppendorf,

Hamburg, Germany

${ }^{3}$ Department of Clinical

Chemistry, University Medical

Center Goettingen, Goettingen, Germany

${ }^{4}$ Laboratory Department 1, Central Institute of the German Armed Forces Medical Services, Koblenz, Germany

${ }^{5}$ Institute for Medical Microbiology, Virology and Hygiene, University Hospital of Rostock, Rostock, Germany ${ }^{6}$ Department of Infectious Disease Epidemiology, Bernard Nocht Institute for Tropical Medicine, Hamburg, Germany ${ }^{7}$ Institute for Medical Microbiology, Virology and Hygiene, University Hospital Eppendorf, Hamburg, Germany

\section{Correspondence to} Lt Col MC Ralf Matthias Hagen, Department of Tropical Medicine at the Bernhard Nocht Institute, German Armed Forces Hospital Hamburg, Bernhard Nocht Street 74 D-20359 Hamburg, Germany; hagen@bni-hamburg.de

Received 20 June 2013 Revised 23 July 2013 Accepted 31 August 2013 Published Online First 10 October 2013

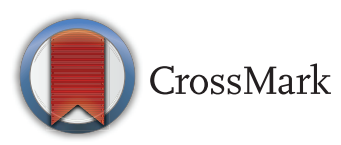

To cite: Hagen RM, Wulff $B$, Loderstaedt $U$, et al. J R Army Med Corps 2014; 160:226-231.

\title{
Is rapid hepatitis $C$ virus testing from corpses a screening option for index persons who have died after mass-casualty incidents in high-prevalence settings in the field?
}

\author{
Ralf M Hagen, ${ }^{1}$ B Wulff, ${ }^{2}$ U Loderstaedt, ${ }^{3}$ I Fengler, ${ }^{4}$ H Frickmann, ${ }^{1,5}$ \\ N G Schwarz, ${ }^{6}$ S Polywka ${ }^{7}$
}

\begin{abstract}
Introduction We tested a commercially available rapid hepatitis C virus (HCV) test assay for its potential use for analyses of corpses as a screening option for index persons who have died after mass-casualty incidents in high-prevalence settings in the field.
\end{abstract}

Materials and methods 50 blood samples were drawn from 16 recently deceased confirmed HCV-positive patients whose corpses were stored at $4^{\circ} \mathrm{C}$ in the mortuary and were analysed at admission and up to $48 \mathrm{~h}$ post mortem by rapid serological testing using the ImmunoFlow HCV test (Core Diagnostics, Birmingham, UK) in comparison with automated serological assays and PCR. Samples from $50 \mathrm{HCV}$-negative corpses were also analysed.

Results The blood of only four of the $16 \mathrm{HCV}$-positive corpses reacted clearly with the ImmunoFlow HCV test, while in five cases the result was only weakly reactive and three cases showed very weak reactivity. Four of the infected corpses showed initially negative results, three of which became very weakly reactive $48 \mathrm{~h}$ post mortem. 49 out of 50 samples (98\%) from HCV-negative corpses tested negative.

Discussion The rapid test system we investigated showed insufficient sensitivity regarding the identification of HCV positivity. Automated serological testing or PCR should be preferred if it is realistically available in the deployed military setting.

\section{INTRODUCTION}

Medical assistance in mass-casualty incidents, for example, in resource-limited areas of the world with high hepatitis $\mathrm{C}$ virus (HCV) prevalence, may lead to direct, unprotected contact with blood from dying injured individuals. In the case of contact of blood with mucous membranes or injured skin, there is considerable risk of $\mathrm{HCV}$ transmission. The early stages of HCV infection are associated with excellent prognosis if treated with interferon- $\alpha$ therapy. ${ }^{1}$ Knowledge of the positive HCV status of a corpse from which infectious material has been inoculated will increase awareness of the need for HCV follow-up for timely identification of potential infection by serology, liver enzyme assessment and quantitative PCR to exclude spontaneous viral clearance. ${ }^{12}$

$\mathrm{HCV}$ is prevalent in virtually all conflict areas of the world, such as in Afghanistan. In the course of 6 years from 2007 to 2012, at the laboratory of the German field camp in Mazar-e Sharif, Afghanistan, HCV antibodies were detected in 1/110 (<1\%) blood samples

\section{Key messages}

- Contact of blood with mucous membranes or injured skin bears considerable risk of hepatitis C virus (HCV) transmission in high-prevalence settings.

- Medical assistance in mass-casualty incidents in resource-limited areas may lead to inoculation of blood of a dying patient.

- HCV testing from dead index patients in the field will usually be based on rapid tests for which evaluation data with blood from corpses are lacking.

- The rapid test system we investigated showed insufficient sensitivity regarding the identification of HCV positivity of corpses.

- Automated serological testing or PCR should be preferred if it is realistically available in theatre.

- Healthcare workers who are inoculated with potentially infective material from dead index persons should be encouraged not to forgo serological follow-up screening because early HCV infections can be treated with excellent prognosis.

from Afghan government officials, in 2/23 (10\%) samples from Afghan civilians who were employed by the German Armed Forces and in 3/145 (2\%) samples of deployed German soldiers (unpublished data).

Sound risk evaluation after blood contact should include reliable HCV test results from the index person; in resource-limited settings these are usually based on rapid tests, which have been validated with blood from living individuals only. There is no experience in the interpretation of the test results if the index person has died prior to sample acquisition. For this reason, we assessed the reliability of a field-compatible rapid HCV test for HCV testing from blood of human corpses at various time points post mortem.

\section{METHODS}

HCV testing using corpse fluids

Samples and sample preparation

Stored blood samples from a previous study ${ }^{3}$ were used in this analysis. They were derived from recently deceased human corpses of 16 known 
$\mathrm{HCV}$-positive and of $50 \mathrm{HCV}$-negative deceased individuals who were donors of corneal and tissue samples. All samples were acquired at the Institute for Forensic Medicine, University Hospital Eppendorf, Hamburg, Germany. The first samples from the HCV-positive patients were taken on their admission to the mortuary and $12 \mathrm{~h}$ post mortem, which was routinely possible if the patients had died at the University Hospital Eppendorf and were sent to the mortuary in time. If $\mathrm{HCV}$ patients had died outside the hospital and there was delay in sending them to the mortuary, the 12 -h sampling could not be done. Subsequent samples were taken at 24,36 and $48 \mathrm{~h}$ post mortem, respectively. Overall, 50 samples from the 16 $\mathrm{HCV}$-positive corpses were tested that were drawn on admission $(\mathrm{n}=5), 12 \mathrm{~h}$ post mortem $(\mathrm{n}=7), 16.5 \mathrm{~h}$ post mortem $(\mathrm{n}=1)$, 18 h post mortem $(n=1), 24$ h post mortem $(n=13), 36$ h post mortem $(n=11)$ and 48 h post mortem $(n=12)$. Samples of 50 $\mathrm{HCV}$-negative patients were acquired without delay after their admission to the mortuary. Subsequent sampling was covered by the ethical clearance for this study for infectious corpses only. Accordingly, only one sample could be obtained from $\mathrm{HCV}$-negative corpses.

Before their arrival at the Institute for Forensic Medicine, the corpses were stored at room temperature $\left(20-25^{\circ} \mathrm{C}\right)$. After arrival at the mortuary, they were stored under standardised conditions at $4^{\circ} \mathrm{C}$ prior to acquisition of subsequent samples.

After disinfection of the sampling site, $20 \mathrm{~mL}$ whole blood was acquired percutaneously through sterile TSK Supra hollow needles $(2.00 \times 100$, TSK Laboratory, Tilburg, The Netherlands) from peripheral large vessels, that is, femoral or subclavian artery and/or vein, and, in rare instances, from the heart if sampling from peripheral vessels failed. The material was subsequently transferred into labelled, empty $10 \mathrm{~mL}$ test tubes (Harre Co., Hannover, Germany). ${ }^{45}$

The more or less haemolysed samples were centrifuged twice at $1000 \mathrm{~g}$ for $10 \mathrm{~min}$ each to separate corpuscles and particles of fat, resulting in a material similar to haemolysed serum. Prior to testing the samples were stored at $-20^{\circ} \mathrm{C}$.

\section{Rapid HCV testing}

ImmunoFlow HCV tests (Core Diagnostics, Birmingham, UK) with a sensitivity of $100 \%$ and a specificity of $99 \%$ for serum (manufacturer's data), which are certified for diagnostic use in Europe, are currently under evaluation for potential use in the deployed operational theatre by the Medical Services of the German Armed Forces and were used for HCV screening with the specimens described above at the Institute for Medical Microbiology, Virology and Hygiene, University Hospital Eppendorf, Hamburg, Germany. In an evaluation study by the US Armed Forces, the test was shown to have a sensitivity of $96.4 \%$ and a specificity of $97.9 \%$ for plasma. However, there was deterioration of sensitivity to $26.2 \%-34.5 \%$ and of specificity to $89.2 \%-98.8 \%$ if whole blood subject to unfavourable storage and temperature conditions was analysed. ${ }^{6}$ Data on the reliability of the ImmunoFlow $\mathrm{HCV}$ test with samples from corpses are not available. The sample material was used as described for serum by the manufacturer.

Samples were considered reactive if they showed a reaction equal to or stronger than the control reaction. A less visible band than the control was considered weakly reactive, and a hardly visible band was defined as a very weak reactivity. If no reaction could be seen, the samples were considered negative.

\section{Confirmation testing and estimation of sample quality}

As the diagnostic gold standard, all 50 samples from $\mathrm{HCV}$-positive patients and the $50 \mathrm{HCV}$-negative samples were tested with the HCV V.3.0 kit on the AXSYM system (Abbott, Wiesbaden-Delkenheim, Germany). The results are depicted as signal/cut-off (s/co) units with a reference value of $<1.00$.

In case of positive HCV results by automated serological testing, determination of HCV load was attempted by the quantitative Cobas Taqman PCR (Roche Diagnostics Deutschland GmbH, Mannheim, Germany) with a detection limit of $15 \mathrm{IU} / \mathrm{mL}$ directly from the sample materials without prior RNA extraction.

To estimate the degree of protein degradation in the samples of the HCV-positive patients, whole-serum protein values (reference value $64-83 \mathrm{~g} / \mathrm{L}$ ) and albumin (reference value $35-50 \mathrm{~g} / \mathrm{L}$ ) were determined (Dimension Vista, Siemens, Berlin and Munich, Germany). For organisational reasons, these analyses could not be performed for all samples from all study patients. $\mathrm{HCV}$-negative corpses were not included in this analysis.

\section{Statistics}

Results for groups of samples from HCV-positive corpses with clearly positive bands, weak bands or very weak bands, and without visible bands in the rapid antibody testing-based ImmunoFlow HCV tests were compared with the s/co unit values of the automated AXSYM system. The distribution of the measured results across the four rapid test categories was displayed using boxplots as a graphical variant of an ANOVA analysis.

\section{RESULTS}

\section{Rapid HCV testing with the ImmunoFlow HCV test}

Altogether 12 analysed samples from 4/16 deceased patients (25\%) known to be HCV-positive and with sampling time points ranging from admission to $48 \mathrm{~h}$ post mortem clearly tested positive for HCV antibodies using the ImmunoFlow rapid test. In five corpses (11 samples), the test was only weakly reactive; one of these patients became clearly positive 24 and $48 \mathrm{~h}$ post mortem. In three of the HCV-positive corpses (seven samples) the rapid test was very weakly reactive; only one of these samples became weakly reactive $36 \mathrm{~h}$ post mortem. The other four HCV-positive corpses (14 samples) tested negative, both initially and at consecutive analyses up to $36 \mathrm{~h}$ post mortem (Figure 1). However, at $48 \mathrm{~h}$ post mortem three out of four of these samples switched from a negative to a very weakly reactive test result. Of note, for three out of the four negatively tested HCV-positive corpses, the infection had been known for $1.5,6$ and 9 years, respectively, excluding weak antibody reactivity due to early seroconversion. The corresponding data were not available for the fourth negatively tested corpse. Among the samples from the HCV-negative corpses, 49 out of 50 tested negative for $\mathrm{HCV}$ antibodies and one sample was weakly reactive.

The control bands of the rapid HCV test appeared in every analysis. Accordingly, false negative results would indeed have been given as negative and not merely as non-interpretable.

\section{Automated serological testing and PCR-based assessment of viral load}

Automated HCV antibody testing on the AXSYM system was positive for all HCV-positive corpses, and negative for all $\mathrm{HCV}$-negative corpses. For the HCV-positive corpses, the mean test values $(\mathrm{s} / \mathrm{co})$ ranged from 3.75 to 125 with a median of 27.5. The quantitative results remained stable within the $48-\mathrm{h}$ analysis period, with most results being scattered within a $\pm 13 \%$ range (median) and $2.9 \%$ and $43.5 \%$ of the respective mean values being the lowest and highest deviations. The mean value $(\mathrm{s} / \mathrm{co})$ of the $\mathrm{HCV}$-negative corpses was 0.1 with an SD of 
Figure 1 Distribution of positive, weakly positive, very weakly positive and negative hepatitis $\mathrm{C}$ virus (HCV) antibody rapid test results from HCV-positive corpses at admission to the mortuary and up to $48 \mathrm{~h}$ post mortem.

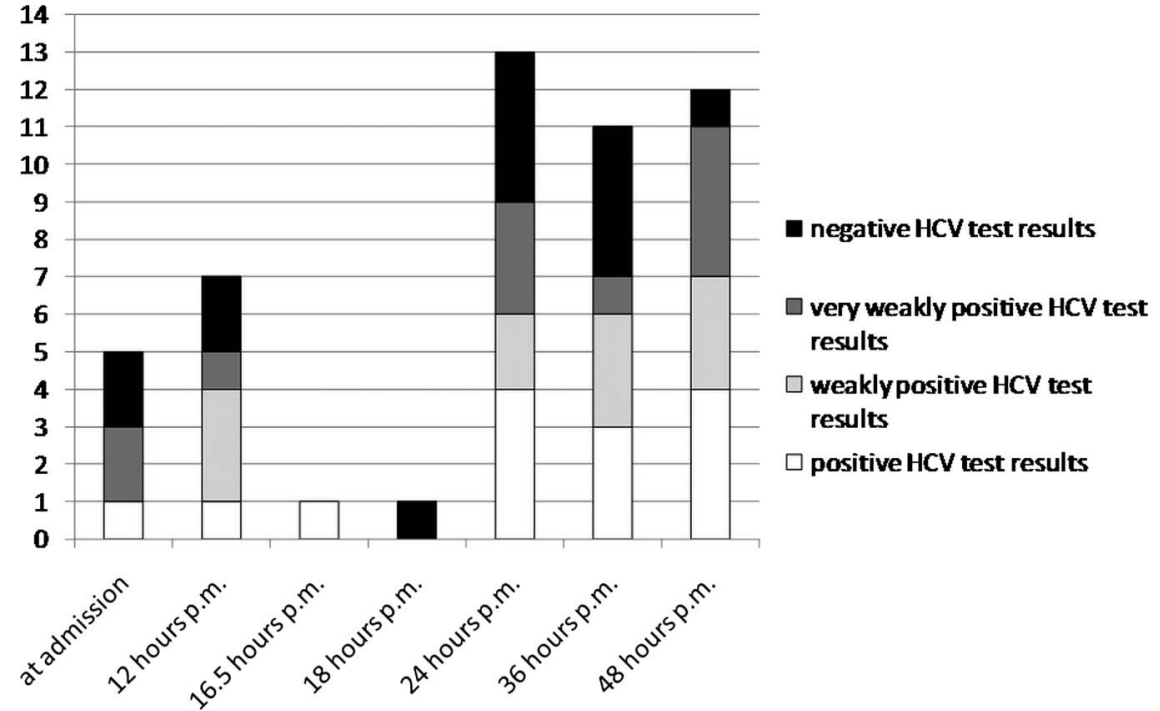

96.3\% and a median of 0.08 . Of note, the negative AXSYM result of the sample scoring falsely positive by rapid testing was confirmed as negative using the ADVIA Centaur HCV assay (Siemens, Munich, Germany) with a test value (s/co) of 0.1 $(<0.8)$.

Positive PCR results were obtained from 10 out of 16 (62.5\%) HCV-positive corpses; all HCV-negative samples remained negative. Switches from positive to negative and vice versa depending on time post mortem were not observed with PCR. Mean values of quantitative PCR were between 1900 and $27500000 \mathrm{IU} / \mathrm{mL}$ for the positively tested samples; the SDs of the measured results at the different postmortem time points ranged from $34.8 \%$ to $91.1 \%$ of the respective mean values, with a median of $63.8 \%$.

\section{Comparison of rapid testing and AXSYM-based testing}

For the measured s/co units of the AXSYM system, there was no difference between the groups of HCV-positive corpses with negative rapid tests and with very weak bands in the rapid tests with mean s/ co values of $20.7( \pm 11.7)$ and $12.0( \pm 11.4)$ ( $p>0.13)$, respectively. In contrast, the groups with weak bands (s/co 45.3 \pm 27.9$)$ and clearly positive rapid tests (s/co 79.3 \pm 39.5 ) differed from each other and from all other groups $(\mathrm{p}<0.05)$. The cut-off for reliable positive results in rapid testing was an s/co value $>40$ of the AXSYM system (Figure 2).

\section{Analysis of protein degradation between admission} and $48 \mathrm{~h}$ post mortem

In 12 out of $15 \mathrm{HCV}$-positive corpses (80\%), the initially observed absolute values were considerably decreased in comparison with reference values from living patients, indicating protein degradation before the corpses were transferred to the mortuary. For whole-serum protein, mean values ranged from 27 to $90 \mathrm{~g} / \mathrm{L}$ (reference 64-83) with a median of $42 \mathrm{~g} / \mathrm{L}$, and for albumin they ranged from 9.2 to $39.5 \mathrm{~g} / \mathrm{L}$ (reference 35-50) with a median of $16.1 \mathrm{~g} / \mathrm{L}$. However, the whole-serum protein as well as albumin remained stable during the storage of the corpses at $4^{\circ} \mathrm{C}$ in the mortuary in the interval between admission and $48 \mathrm{~h}$ post mortem. For whole-serum protein, the SDs of the measured results at the different post-mortem time points ranged from $2.6 \%$ to $30.7 \%$ of the respective mean values with a median of $8.5 \%$; for albumin, the SD ranged from $0 \%$ to $18.0 \%$ of the mean values with a median of $6.3 \%$. These data reflect the stable results in antigen/antibody-based (and thus ultimately protein-based) HCV testing on the AXSYM system.

\section{DISCUSSION}

In mass-casualty care, such as in humanitarian aid missions, contact with blood and its incorporation via mucous membranes or needle-stick injuries is likely. The risk of HCV acquisition from an infected index person in case of needle-stick injuries is about $3 \%$ and may be higher in case of high viral load. ${ }^{7-9}$ The yearly number of professional HCV infections worldwide is estimated to be $16000 .^{10}$ There is considerable risk of HCV transmission in countries with high endemicity. Humanitarian aid missions in conflict areas with a high HCV burden are associated with an increased risk. In asymmetric military conflicts, there is the additional jeopardy of infection due to ballistic

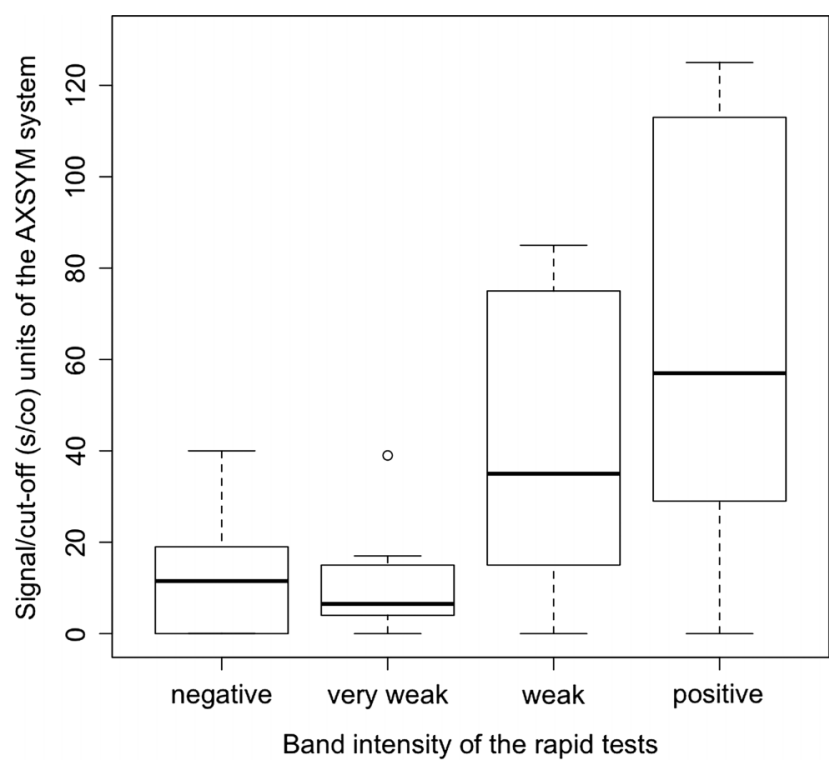

Figure 2 Signal/cut-off units (s/co) of the AXSYM system in samples with negative, very weakly positive, weakly positive and positive rapid hepatitis $C$ virus test results. Dot=outlier. Heavy horizontal lines=mean values. Boxes=left- or right-shifted distributions around the mean value. SD is indicated above and below the boxes. Cut-off of the AXSYM system: <1.0 s/co. 
transmission via bone fragment implantation during suicide bombing. ${ }^{5}$ For example, in Afghanistan, HCV seroprevalence of $36.6 \%$ among injecting drug users ${ }^{11}$ provides an easily accessible source for the self-inoculation of potential suicide assassins with fresh, replicative virus-containing blood.

Post-exposure measures after accidental inoculation of replicative virus are currently not available for $\mathrm{HCV}$ infection. However, the course of the disease can be significantly affected by therapeutic measures, particularly if the infection is detected at an early stage. If treatment is started at an early stage of infection, interferon- $\alpha$ sole therapy can lead to resolution rates up to $90 \%$ or even higher. ${ }^{1}$ In chronic infections, favourable outcomes are still common, but the therapeutic schemes are more complex ${ }^{1}$ and therapeutic success is at least partially dependent on the virus subtype ${ }^{12}$ and the Il-28B alleles of the infected individual. $^{13}$

Early identification of an HCV infection largely depends on the awareness that a potential transmission event might have occurred. The long interval of several months between the transmission event and final seroconversion ${ }^{14}$ makes it difficult to recall every potentially relevant incident.

Compliance with post-exposure screening procedures after needle-stick injuries and related accidents is generally low, as was recently clearly shown for a German University Hospital, ${ }^{15}$ especially regarding the important follow-up screenings. Failure to consider the possibility that a particular index patient might be infected is the most typical reason for this non-compliance. ${ }^{15}$

It is therefore desirable to have reliable HCV antibody test results from the index patient to increase the awareness of the risk to the injured in case of positive test results. In resourcelimited settings, such screenings have to be based upon rapid screening tests. Rapid HCV antibody tests were shown to have varying sensitivities and specificities, both showing a wide range from $70 \%$ to $100 \%$ in various studies, ${ }^{6}{ }^{16-22}$ with a further decline in reliability when pre-analytic conditions were poor. ${ }^{6} 23$

In detail, the results seem to depend on the test system ${ }^{6}$ and the test setting. ${ }^{23}$ Particularly in high-risk settings such as Uganda and India, rapid HCV testing shows unreliable results in comparison with traditional enzyme immunoassays and PCR. ${ }^{17}{ }^{24}$ Rapid HCV testing of blood donors in rural Cambodia and Vietnam showed a sensitivity as low as $86.6 \%$, an unacceptably poor result for blood donor screening. ${ }^{25}$ Both poor pre-analytic conditions $^{623}$ and HIV positivity ${ }^{1626}$ can lead to declines in the sensitivity of rapid HCV test systems. Specificity may be poor as well, as demonstrated by a Pakistani study showing $2.35 \%$ falsely positive anti-HCV test results from blood with an immunochromatographic device. ${ }^{27}$

The best results from rapid tests are usually achieved in standardised evaluation studies, leading to sensitivities and specificities $>98 \%,{ }^{22} 2829$ even for rapid multiplexed immunoassays (MBio Diagnostics, Boulder, Colorado, USA), ${ }^{30}$ and for various sample materials such as venous blood, finger-stick blood, serum, plasma or oral fluid (OraSure Technologies, Inc., Bethlehem, Pennsylvania, USA). ${ }^{31}$ The OraQuick HCV test (OraSure Technologies, Inc.) in particular, suitable for blood and oral fluid, scored well in various evaluation studies. ${ }^{6} 18$ 31-33 In contrast, the rapid chromatographic Toyo anti-HCV test (Toyo Diagnostics UK, London, UK) showed a poor specificity of $88 \%$ in spite of a sensitivity of $99 \%$ for serum and EDTA blood. ${ }^{34}$ The sensitivity of the BioRapid HCV test (biorapid GmbH, Umkirch, Germany) on donor plasma was only $84 \% .^{6}$ The Chembio finger-stick blood, Chembio oral fluid (Chembio Diagnostics, Inc., Medford, New York, USA) and MedMira (MedMira Inc., Halifax, Canada) finger-stick blood tests achieved sensitivities of $87.1 \%, 85.4 \%$ and
$80.0 \%$ and specificities of $99.0 \%, 100 \%$ and $100 \%$ with serum, respectively. ${ }^{20}$ In another study, Chembio and MedMira rapid tests showed sensitivities of $96.2 \%-98 \%$ and $86.8 \%-88.3 \%$ and specificities $>99.5 \%$ with serum, respectively, while OraSure tests showed sensitivities and specificities of 97.8\%-99.3\% and $>99.5 \%$, respectively. ${ }^{26}$ Another study suggested sensitivity of $98 \%$ and specificity of $100 \%$ with serum for the MedMira rapid technique. ${ }^{35}$ Sensitivity and specificity of the SM-HCV rapid tests (SERO-med Laborspezialitäten GmbH, Dollnstein, Germany) with serum were $98 \%$ and $100 \%$, respectively. ${ }^{36}$ The rapid HCV diagnostic kit by J. Mitra Co. (New Delhi, India) exhibited sensitivity of $87.5 \%$ and specificity of $100 \%$ with serum. ${ }^{37}$ It is evident that the reliability of various HCV rapid test systems is variable.

If the index patient dies before blood samples can be acquired, for example, in the course of a mass-casualty event, corpse blood has to be analysed. Because no data on the reliability of rapid HCV screenings from corpse blood are available, we tested the ImmunoFlow HCV test that is under evaluation by the German Armed Forces Medical Service with blood of HCV-positive and HCV-negative human corpses.

We demonstrated that rapid HCV antibody testing is hampered by poor sensitivity, since only four of the HCV-positive corpses were clearly reactive, and five showed a weakly positive result. Low s/co values in automated testing were significantly associated with failure of rapid testing. The relevance of a very weak reactivity obtained in three of the HCV infected corpses is unknown since it was also found in $3 / 4 \mathrm{HCV}$-infected patients who tested negative until the final sample was drawn $48 \mathrm{~h}$ post mortem. Thus, these very weak positive results might have been due to unspecific binding of components of the haemolysed blood to the matrix. Rapid sample acquisition might reduce the risk of such non-specific results, but with a negative test result HCV infection of the index person cannot be excluded and the exposed persons should be encouraged to be re-tested to rule out seroconversion. As expected, because of the lack of antigen component in the rapid test we assessed, there was no statistical difference between samples with high or low HCV copy numbers in PCR regarding their rapid test results (data not shown). Accordingly, high infectivity can be associated with negative rapid test results.

It is a major limitation of this study that no subsequent samples could be taken from the HCV-negative samples because there was no ethical clearance for this. The question whether progressive decay might have led to a higher rate of false reactivity remains unanswered. However, even in a masscasualty setting, sample acquisition from deceased index patients should be possible within several hours, corresponding to the estimated average transport time of the HCV-negative corpses to the mortuary in our study.

It is theoretically possible that HCV rapid test kits that scored better with whole blood samples after unfavourable transport and temperature conditions ${ }^{6}$ might have led to better results with corpse blood as well. However, the limited quantities of sample did not allow for broad evaluation studies, so only the test system that is under evaluation by the German Armed Forces Medical Service was assessed to identify potential diagnostic pitfalls of corpse blood analyses by HCV antibody rapid tests.

Automated serological testing scored best concerning reliable discrimination of $\mathrm{HCV}$-positive and $\mathrm{HCV}$-negative corpses as described earlier, ${ }^{4}$ but it is unlikely to be available in the field or in resource-limited areas. The same is true for testing for HCV RNA by PCR, which has previously been shown to be possible even up to 48 h post mortem. ${ }^{3}$ Of the $16 \mathrm{HCV}$-positive corpses, 
10 were shown to be viraemic throughout follow-up, while six had negative PCR results due either to low viral replication or to cured infection or inhibitory effects of the haemolysed blood.

The observed stability of protein content in the corpse blood during $48 \mathrm{~h}$ post mortem may be attributed to the storage of the bodies at $4^{\circ} \mathrm{C}$. If corpses are kept in a tropical environment, protein degradation might be much faster. Basic sample preparation is advisable. If no centrifugation is possible, at least sedimentation of serum samples should be aimed for.

Finally, the interpretation should consider the time-window of serological HCV screenings and the associated risk of infections from antibody-negative $\mathrm{HCV}$-infected index persons ${ }^{38} 39$ if early infection of the dead index persons seems likely. Regarding seroconversion sensitivity, the ImmunoFlow HCV test was $0.4-5$ days less sensitive than $7 / 9$ recently compared automated and classical HCV antibody tests in a recent analysis by a commercial assessment service ${ }^{40}$ (kindly provided by mölab $\mathrm{GmbH}$, Langenfeld, Germany). Field-compatible HCV antigen tests ${ }^{41} 42$ might be considered in cases of suspected early infection, but their suitability for diagnostic use with corpse material is still to be demonstrated.

\section{CONCLUSIONS}

Rapid HCV antibody testing of samples from corpses under field conditions based on the test system investigated here is associated with poor sensitivity and with specificity that depends on time post mortem. Accordingly, results should be interpreted with care, particularly considering the postmortem time point of sample acquisition and the time-window in cases of an early HCV infection of the index patient. Automated serological testing should be preferred if it is realistically available in the circumstances.

Acknowledgements The authors thank Sven Helfer for excellent technical assistance and are grateful to Florian Helm for extracting the data on HCV detections in the German field camp in Mazar-e Sharif, Afghanistan, from the local database.

Contributors Guarantor: RMH. Planning: RMH, BW, UL, IF, HF, NGS and SP Conduct: HF, NGS and SP. Reporting: RMH, BW, UL, IF, HF, NGS and SP.

Funding The HCV rapid testing was funded by the German Ministry of Defense (MoD), scientific project ('Sonderforschungsprojekt') 'Optimisation of microbiological diagnostic pre-analytics under tropical conditions' (15K2-S-451315).

Competing interests None.

Ethics approval Ethical clearance was obtained from the Ethics Committee of the Medical Association of Hamburg, Germany (WF-024/09). The Ethics Committee granted permission in the absence of explicit consent by the deceased. Written consent was obtained from the relatives of the deceased.

Provenance and peer review Not commissioned; externally peer reviewed. Data sharing statement All available data are presented in the manuscript.

\section{REFERENCES}

1 European Association for the Study of the Liver. EASL Clinical Practice Guidelines: management of hepatitis C virus infection. J Hepatol 2011;55:245-64.

2 Hofer $\mathrm{H}$, Watkins-Riedel T, Janata 0 , et al. Spontaneous viral clearance in patients with acute hepatitis C: predictability by repeated measurements of serum HCV concentration. Hepatology 2002;36:286A.

3 Meyer T, Polywka S, Wulff B, et al. Virus NAT for HIV, HBV, and HCV in post-mortal blood specimens over $48 \mathrm{~h}$ after death of infected patients-first results. Transfus Med Hemother 2012:39:376-80.

4 Edler C, Wulff B, Schröder AS, et al. A prospective time-course study on serological testing for human immunodeficiency virus, hepatitis $B$ virus and hepatitis $C$ virus with blood samples taken up to $48 \mathrm{~h}$ after death. J Med Microbiol 2011;60: 920-6.

5 Frickmann $\mathrm{H}$, Wulff $\mathrm{B}$, Loderstaedt U, et al. From IEDs to AIDS? Detection of HIV in human corpses by rapid screening tests after suspected intentional transmission in terrorist attacks. J R Army Med Corps 2013:159:278-82.
6 O'Connel RJ, Gates RG, Bautista CT, et al. Laboratory evaluation of rapid test kits to detect hepatitis $C$ antibody for use in predonation screening in emergency settings. Transfusion 2013:53:505-17.

7 Trim JC, Elliot TS. A review of sharps injuries and preventative strategies. J Hosp Infect 2003:53:237-42.

8 Hanrahan A, Reutter L. A critical review of the literature on sharps injuries: epidemiology, management of exposures and preventions. J Adv Nurs 1997:1:144-54

9 Yazdanpanah Y, De Carli G, Migueres B, et al. Risk factors for hepatitis C virus transmission to health care workers after occupational exposure: a European case-control study. Clin Infect Dis 2005:41:1423-30.

10 Prüss-Üstün A, Rapiti E, Hutin Y. Estimation of the global burden of disease attributable to contaminated sharps injuries among healthcare workers. Am J Ind Med 2005:48:482-90

11 Nasir A, Todd CS, Stanekzai MR, et al. Implications of hepatitis C viremia vs. antibody alone on transmission among male injecting drug users in three Afghan cities. Int J Infect Dis 2011:15:e201-5.

12 Cooreman MP, Schoondermark-Van de Ven EM. Hepatitis C virus: biological and clinical consequences of genetic heterogeneity. Scand I Gastroentero/ Supp/ 1996:218:106-15.

13 Ge D, Fellay J, Thompson AJ, et al. Genetic variation in IL28B predicts hepatitis C treatment-induced viral clearance. Nature 2009:461:399-401.

14 Van der Poel CL, Cuypers HT, Reesink HW. Hepatitis C virus six years on. Lancet 1994:344:1475-9.

15 Frickmann $H$, Reisinger $E$, Mittlmeier $T$, et al. Prophylaxis against infections after needle stick injuries. Unfallchirurg 2012;115:708-16 [Article in German].

16 Desbois D, Vaghefi P, Savary J, et al. Sensitivity of a rapid immuno-chromatographic test for hepatitis C antibodies detection. J Clin Virol 2008:41:129-33.

17 Seremba E, Ocama P, Opio CK, et al. Poor performance of hepatitis C antibody tests in hospital patients in Uganda. J Med Virol 2010;82:1371-8.

18 Lee SR, Kardos KW, Schiff E, et al. Evaluation of a new, rapid test for detecting HCV infection, suitable for use with blood or oral fluid. J Virol Methods 2011;172:27-31.

19 Ivantes CA, Silva D, Messias-Reason I. High prevalence of hepatitis C associated with familial history of hepatitis in a small town of south Brazil: efficiency of the rapid test for epidemiological survey. Braz J Infect Dis 2010;14:483-8.

20 Jewett A, Smith BD, Garfein RS, et al. Field-based performance of three pre-market rapid hepatitis $C$ virus antibody assays in STAHR (Study to Assess Hepatitis C Risk) among young adults who inject drugs in San Diego, CA. J Clin Virol 2012:54:213-7

21 Souza IO, Schaer RE, Lima FW, et al. Validation of an immunochromatographic assay for the multiple detection of specific antibodies against HIV, HBV and HCV. Clin Vaccine Immunol 2013:20:128.

22 Xiang $T$, Jiang Z, Zheng J, et al. A novel double antibody sandwich-lateral flow immunoassay for the rapid and simple detection of hepatitis C virus. Int J Mol Med 2012;30:1041-7.

23 Bienek DR, Charlton DG. The effect of simulated field storage conditions on the accuracy of rapid user-friendly blood pathogen detection kits. Mil Med 2012:177:583-8

24 Firdaus R, Saha K, Sadhukhan PC. Rapid immunoassay alone is insufficient for the detection of hepatitis C virus infection among high-risk population. J Viral Hepat 2013:20:290-3.

25 Bjoerkvoll B, Viet L, Ol HS, et al. Screening test accuracy among potential blood donors of $\mathrm{HBsAg}$, anti-HBC and anti-HCV to detect hepatitis $B$ and $C$ virus infection in rural Cambodia and Vietnam. Southeast Asian J Trop Med Public Health 2010:41:1127-35.

26 Smith BD, Drobeniuc J, Jewett $A$, et al. Evaluation of three rapid screening assays for detection of antibodies to hepatitis C virus. J Infect Dis 2011;204:825-31.

27 Batool A, Khan MI, Bano KA. Efficacy of immunoassay chromatography test for hepatitis-C antibodies detection. J Ayub Med Coll Abbottabad 2009;21:38-9.

28 Montebugnoli L, Borea G, Miniero R, et al. A rapid test for the visual detection of anti-hepatitis C virus antibodies in whole blood. Clin Chim Acta 1999;288:91-6.

29 Daniel HD, Abraham P, Raghuraman $\mathrm{S}$, et al. Evaluation of a rapid assay as an alternative to conventional enzyme immunoassays for detection of hepatitis $C$ virus-specific antibodies. J Clin Microbiol 2005;43:1977-8.

30 Lochhead MJ, Todorof K, Delaney M, et al. Rapid multiplexed immunoassay for simultaneous serodiagnosis of HIV-1 and coinfections. J Clin Microbiol 2011:49:3584-90.

31 Lee SR, Yearwood GD, Guillon GB, et al. Evaluation of a rapid, point-of-care test device for the diagnosis of hepatitis C infection. J Clin Virol 2010;48:15-17.

32 Drobnik A, Judd C, Banach D, et al. Public health implications of rapid hepatitis C screening with an oral swab for community-based organizations serving high-risk populations. Am J Public Health 2011;101:2151-5.

33 Cha YJ, Park Q, Kang ES, et al. Performance evaluation of the OraQuick hepatitis C virus rapid antibody test. Ann Lab Med 2013;33:184-9.

34 Kant J, Möller B, Heyne R, et al. Evaluation of a rapid on-site anti-HCV test as a screening tool for hepatitis C virus infection. Eur J Gastroenterol Hepatol 2013:25:416-20

35 Buti M, Cotrina M, Chan $\mathrm{H}$, et al. Rapid method for the detection of anti-HCV antibodies in patients with chronic hepatitis C. Rev Esp Enferm Dig 2000;92:140-6. 
36 Yuen MF, Hui CK, Yuen JC, et al. The accuracy of SM-HCV rapid test for the detection of antibody to hepatitis C virus. Am J Gastroenterol 2001;96:838-41.

37 Kaur H, Dhanao J, Oberoi A. Evaluation of rapid kits for detection of HIV, HBsAg and HCV infections. Indian J Med Sci 2000;54:432-4.

38 Tugwell BD, Patel PR, Williams IT, et al. Transmission of hepatitis $\mathrm{C}$ virus to several organ and tissue recipients from an antibody-negative donor. Ann Intern Med 2005;143:648-54.

39 Kucirka LM, Sarathy H, Govindan P, et al. Risk of window period hepatitis-C infection in high infectious risk donors: systematic review and meta-analysis. Am J Transplant 2011;11:1188-200.
40 Gonen G, Perry K. An assessment of the seroconversion sensitivity of ImmunoFlow HCVrapid test device (Core Diagnostics). Report PER 06010. London:

Microbiological Diagnostics Assessment Service, Evaluations and Standards Laboratory, Health-Protection Agency—Centre for Infections, 2-7.

41 Attallah AM, Ismail H, Tabll AA, et al. A novel antigen detection immunoassay for field diagnosis of hepatitis C virus infection. J Immunoassay Immunochem 2003;24:395-407.

42 Mikawa AY, Santos SA, Kenfe FR, et al. Development of a rapid one-step immunochromatographic assay for HCV core antigen detection. J Virol Methods 2009;158:160-4 